\title{
INTERFEROMETRY AND DISSIPATIVE OPTICS WITH ATOMS
}

\author{
P. SzRiftgiser*, D. Guéry-Odelin, P. Desbiolles, J. Dalibard \\ Laboratoire Kastler Brossel, 24 rue Lhomond, 75005 Paris, France
}

\author{
M. ARNDT \\ Institut für Experimentalphysik der Universität Innsbruck \\ Technikerstr. 25, 6020 Innsbruck, Austria \\ and A. Steane \\ Clarendon Laboratory, Oxford, OX13PU, United Kingdom
}

\begin{abstract}
We have designed a gravitational cavity for ultra-cold atoms using an atomic mirror made from an evanescent laser wave. By a temporal variation of the evanescent wave intensity, we have realized various atom optics components such as temporal slits and phase modulators. We have also designed an atom interferometer using this cavity which proves that the coherence of the de Broglie waves can be preserved during the bounce of the atoms on the mirror. Finally we show that an efficient cooling of the atoms inside the cavity can be achieved using a Sisyphus process during the bounce.
\end{abstract}

PACS numbers: $03.75 . \mathrm{Dg}, 32.80 . \mathrm{Pj}, 42.50 . \mathrm{Vk}$

\section{Introduction}

In textbook presentations of quantum mechanics, a central argument for the validity of the theory is the equivalence of the behavior of material particles and light in a given interferometric setup, such as a Young slit or a Bragg diffraction experiment. From this equivalence we deduce the wave nature of particles, the optical wavelength being replaced by the de Broglie wavelength in the expressions for the fringe spacing or the diffraction angles.

The central point of this paper is to address the directly related question: are there situations where atoms and photons will give qualitatively different results in a given interference experiment? For steady-state problems the answer is negative since equal wavelength for light and matter will produce equal diffraction patterns. On the contrary, for time dependent problems, the dispersion relation

*Present address: Laboratoire de Spectroscopie Hertzienne de Lille, Bâtiment P5, 59655 Villeneuve d'Ascq, France. 
enters into play and it becomes possible to investigate the nature of the interfering or diffracting objects.

Consider for example the following "Gedanken" experiment where a quasi-monochromatic wave packet is incident on a chopper (Fig. 1). The chopper is opened only for two short periods of duration $\tau$ and one looks for the signal on a detector located after the chopper. Is there any interference observable on this detector? In the case of photons propagating in vacuum, the answer is negative since the two wave packets will not spread and they will never overlap. On the contrary, for nonrelativistic material particles, the dispersion relation is not linear and the spreading of the two wave packets may be large enough so that they overlap onto the detector: an interference signal may be observed.

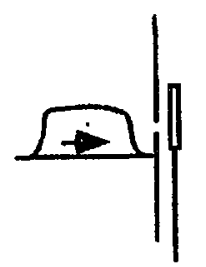

(a)

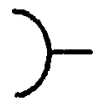

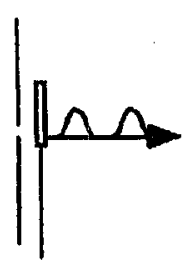

(b)

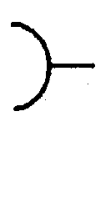

Fig. 1. (a) A quasi-monochromatic wave packet is incident onto a chopper which is opened for two short periods. (b) For matter waves the spreading of the two wave packets may lead to interferences in the signal recorded by the detector. This is not possible for light waves propagating in a non dispersive medium.

We present in this paper experiments performed with cesium atoms and addressing this class of time-dependent problems in quantum mechanics. We first describe the basic tool which allows us to chop and modulate atomic de Broglie waves, i.e. the atomic mirror (Sec. 2). We then present an observation of the time-diffraction phenomenon mentioned above (Sec. 3). In Sec. 4 we show that it is indeed possible to observe interferences between de Broglie waves by letting two wave packets spread and overlap. We then describe in Sec. 5 a phase modulator for atomic de Broglie waves. Finally we present in Sec. 6 a side experiment showing that an evanescent wave mirror can also provide an efficient cooling of the bouncing atoms using a Sisyphus process.

\section{The atomic mirror}

The key element for all the experiments presented in this paper is an atomic mirror formed by an evanescent light wave extending from a glass surface into the vacuum $[1,2]$. The electric field in this wave gives rise to a potential for the atom which is simply the light shift of the atomic ground state

$$
U=\hbar \Omega^{2} / 4 \delta, \quad \text { for }|\delta| \gg \Omega,
$$

$\Omega=d E / 2 \hbar$ is the Rabi frequency in the evanescent wave, proportional to the electric field amplitude $E$ and the atomic dipole moment $d ; \delta=\omega_{\mathrm{L}}-\omega_{\mathrm{A}}$ is the detuning between the laser frequency $\omega_{\mathrm{L}}$ and the atomic resonance frequency $\omega_{\mathrm{A}}$. The electric field falls off with a distance $z$ into the vacuum as $\exp (-\kappa z)$, where 
the characteristic distance $\kappa^{-1} \approx 0.19 \mu \mathrm{m}$. The potential is repulsive at positive detunings providing thus the desired mirror.

The prism surface is concave, with a radius of curvature $2 \mathrm{~cm}$. The evanescent wave (EW) has a circular Gaussian profile, with $1 / e^{2}$ radius $w=300 \mu \mathrm{m}$. It is formed using the total internal reflection of a laser with intensity $I=100 \mathrm{~mW}$, with a linear polarization in the reflection plane; it is blue-detuned by $\delta / 2 \pi=$ 1 to $10 \mathrm{GHz}$ from the resonance line $6 s_{1 / 2}, F=4 \leftrightarrow 6 p_{3 / 2}, F=5$. The EW therefore creates a potential barrier along the $z$-vertical direction $U(x, y, z) \propto$ $I \exp \left[-2\left(x^{2}+y^{2}\right) / w^{2}\right] \exp (-2 \kappa z) / \delta$. The incident atomic velocity on the mirror is $v_{i}=25 \mathrm{~cm} / \mathrm{s}$, corresponding to a free fall of $3 \mathrm{~mm}$ (see below). The $\mathrm{EW}$ mirror is switched on and off by an acousto-optic modulator used in the zeroth order, triggered by a quartz-stabilized function generator. The switching time is $\approx 0.5 \mu \mathrm{s}$, similar to the atomic bouncing time $1 / \kappa v_{i} \approx 1 \mu \mathrm{s}$.

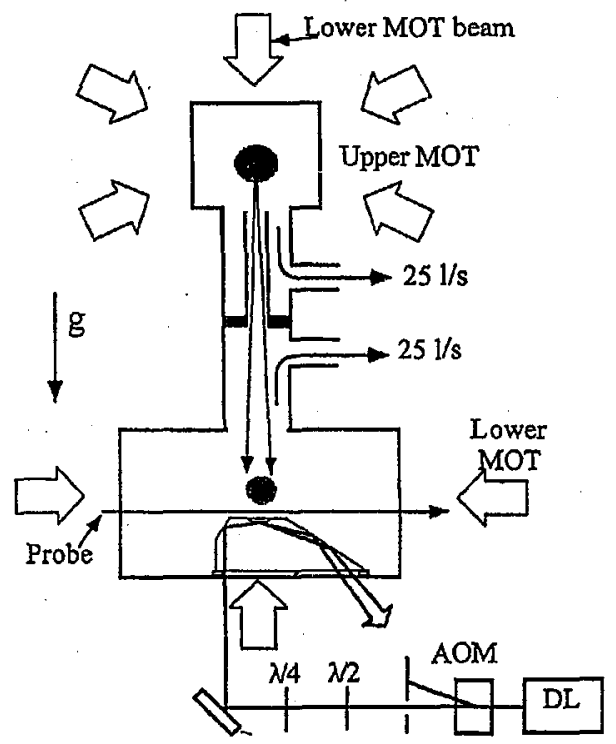

Fig. 2. Experimental setup. Atoms are captured in the upper MOT from a cesium vapor, and transferred to the lower MOT in a high vacuum cell. They are then cooled and dropped into the evanescent wave mirror, which is formed using light produced by a diode laser and which may be time modulated by an acousto-optic modulator.

Our vacuum system is based on two glass cubes of side $10 \mathrm{~cm}$, one positioned $70 \mathrm{~cm}$ above the other (see Fig. 2). Each is evacuated by a $25 \mathrm{l} / \mathrm{s}$ ion pump, and they are connected through a narrow glass tube $(\phi 9 \mathrm{~mm}$, length $140 \mathrm{~mm})$ to allow differential pumping. This system allows us to produce a good vacuum in the lower cell $\left(\leq 3 \times 10^{-9} \mathrm{mbar}\right)$ while having sufficient vapor pressure of cesium in the upper cell $\left(6 \times 10^{-8} \mathrm{mbar}\right)$ to load a magneto-optical trap (MOT) there in a short time.

The experimental cycle begins by loading $3 \times 10^{8}$ atoms into the MOT in the upper cell during 1 second. The collected atoms are then cooled to $5 \mu \mathrm{K}$ in optical 
molasses: the laser intensity is switched to $1 \mathrm{~mW} / \mathrm{cm}^{2}$ per beam, the magnetic field gradient is cut and the detuning $\delta$ between the laser and atomic frequencies is ramped to $\delta=-9 \Gamma$, where $\Gamma=2 \pi \times 5.3 \mathrm{MHz}$ is the FWHM of the atomic resonance. The trap light is then switched far from the atomic resonance, and the atoms fall into the lower chamber.

After a free fall time of $365 \mathrm{~ms}$, a MOT in the lower cell is switched on for $100 \mathrm{~ms}$. The overall transfer efficiency is about $20 \%$, so this provides $6 \times 10^{7}$ atoms each 1.4 seconds, with a lower trap lifetime $\approx 12 \mathrm{~s}$. Once the atoms are caught in the lower MOT, they are compressed to a density $5 \times 10^{10} \mathrm{~cm}^{-3}$ by reducing the laser intensity in the MOT to $0.5 \mathrm{~mW} / \mathrm{cm}^{2}$ at $\delta=-3 \Gamma$ during $3 \mathrm{~ms}$, and then cooled to $3.6 \mu \mathrm{K}$ at $\delta=-9 \Gamma$ in optical molasses during $20 \mathrm{~ms}$. The center of the lower MOT is located $3 \mathrm{~mm}$ above the surface of the prism where the EW forms the mirror. The atoms are then dropped and hit the mirror $\approx 25 \mathrm{~ms}$ later; multiple bounces have been observed in this gravitational cavity [3].

\section{Temporal diffraction of de Broglie waves}

When a beam of particles with a well-defined energy is chopped into a short pulse, the outcoming energy distribution is broadened according to the time-energy uncertainty relation. This effect is very well known for photons and it is at the basis of spectroscopy with ultra-short pulses of light. For matter waves, the phenomenon of diffraction by a time slit has been studied theoretically by several authors [4].

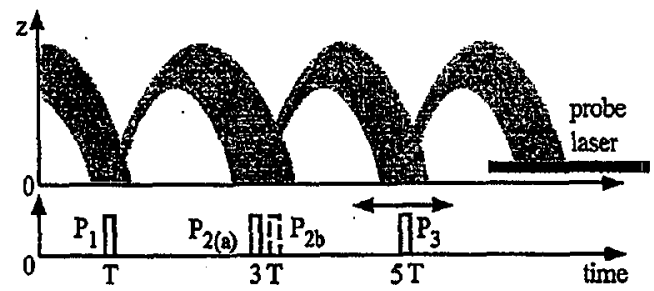

Fig. 3. A cold atomic cloud is released above a mirror formed by an evanescent laser wave. The mirror intensity $I$ (lower curve) is chopped for two short pulses $P_{1}$ and $P_{2}$. The energy distribution is probed using a time-of-flight method, by measuring the number of atoms bouncing on a third pulse $P_{3}$ whose temporal position is scanned. By replacing $P_{2}$ by two pulses $P_{2 \mathrm{a}}$ and $P_{2 \mathrm{~b}}$ (dashed line), we realize the temporal equivalent of a Young slit interferometer.

To investigate experimentally this effect [5], we start at $t=0$ with the atomic cloud from the MOT released above the atomic mirror. We first select atoms with a well-defined total (kinetic+potential) energy using a sequence of two bounces (Fig. 3), generated by chopping the atomic mirror on for two short pulses $P_{1}$ and $P_{2}$; these pulses are respectively centered at time $T=25 \mathrm{~ms}$ and $3 T$, with a variable width $\tau$. From classical mechanics, one would expect after $P_{2}$ a triangular energy distribution centered on $E_{0}=m g^{2} T^{2} / 2=h \times 10 \mathrm{MHz}$ ( $m$ is the atomic mass and $g=9.81 \mathrm{~m} / \mathrm{s}^{2}$ ), with a full width at half maximum (FWHM) $\Delta E_{\mathrm{cl}}=E_{0} \tau / T$. This prediction has to be compared with the typical width $\Delta E_{\mathrm{Qu}}=h / \tau$ of the 
sine-shape energy distribution obtained after the diffraction of a monoenergetic beam by the pulse $P_{2}$. The range at which diffraction phenomenons become predominant corresponds to $\Delta E_{\mathrm{Qu}} \geq \Delta E_{\mathrm{cl}}$, leading to $\tau \leq 50 \mu \mathrm{s}$.

The energy distribution after $P_{2}$ is determined by a time-of-flight technique, by measuring the number of atoms bouncing on a third pulse $P_{3}$, whose temporal position is scanned around $5 T$.

The width of $P_{3}$ is also $\tau$, so that the broadening of the energy distribution in the measurement process remains small. The number of atoms which can perform this sequence of three bounces varies as $\tau^{2}$ in the classical region $(\tau>50 \mu \mathrm{s})$ and as $\tau^{3}$ in the quantum region, provided $P_{3}$ is centered on $5 T$. To increase this number, we have used for $P_{1}, P_{2}$ and $P_{3}$ a grating of pulses instead of a single pulse. The separation between two consecutive pulses of the grating ranges from $400 \mu \mathrm{s}$ for $\tau \leq 40 \mu \mathrm{s}$, up to $1200 \mu \mathrm{s}$ for $\tau=100 \mu \mathrm{s}$; it is chosen large enough so that there is no overlap between the various patterns going through the time slits. The number of atoms per shot for $\tau=40 \mu \mathrm{s}$ is $\approx 40$ for the optimal position of the grating of pulses $P_{3}$.

In order to detect those atoms, we have measured the fluorescence induced by a weak resonant probe beam using a cooled photomultiplier. Each atom scatters $\approx 10^{4}$ photons, among which 10 are detected on average, due to the detection solid angle and to the photomultiplier sensitivity. The signal therefore constitutes in a bunch of $40 \times 10=400$ photons, distributed over the duration ( $4 \mathrm{~ms})$ of the detection window. The stray light is responsible for a similar background signal $\left(10^{5}\right.$ detected photons/s). We note that this detection scheme was possible thanks to the double cell system, which guarantees that the Cs vapor pressure is low in the prism region and that the resonant scattering from this vapor remains small enough.

A typical time-of-flight spectrum is presented in Fig. 4a for $\tau=30 \mu$ s. It gives the fluorescence induced by the probe laser as the temporal position of $P_{3}$ is
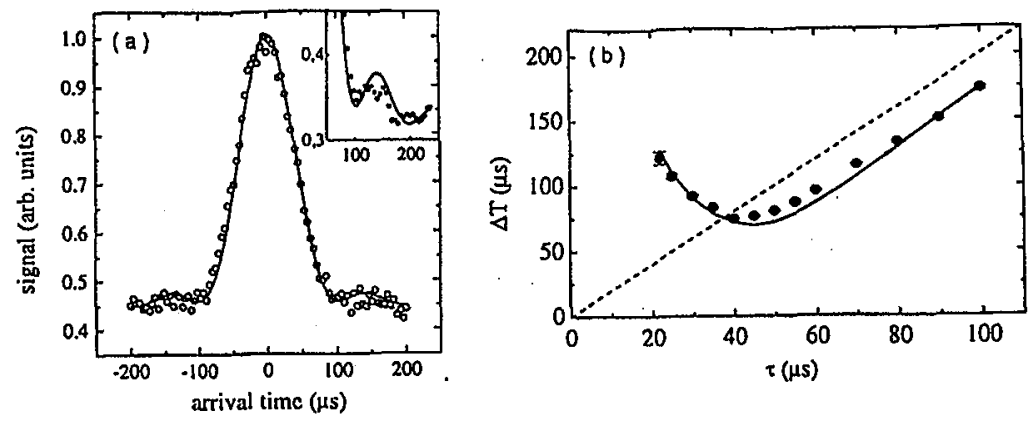

Fig. 4. (a) Circles - experimental time-of-flight signal for a pulse duration $\tau=$ $30 \mu \mathrm{s}$; continuous line - theoretical prediction using the temporal version of the Huygens-Fresnel principle. Inset - magnification of the side band signal. (b) • and continuous line - experimental and theoretical FWHM $\Delta T$ of the time-of-flight signals, as a function of the pulse width $\tau$. Dotted line - classical prediction $\Delta T=2 \tau$. 
scanned. Each of the 90 points of this figure has been averaged 170 times so that the total acquisition time for such a plot is 7 hours. We have repeated this experiment for various $\tau$ and we have plotted in Fig. $4 \mathrm{~b}$ the corresponding FWHM $\Delta T$. For large $\tau$, we find that $\Delta T$ increases with $\tau$ as expected from classical mechanics. When $\tau$ decreases, $\Delta T$ passes through a minimum and increases again, as expecied from the time-energy uncertainty relation. The error bars shown in Fig. 4b indicate the statistical fluctuations of $\Delta T$ in a series of measurements. We have also plotted in Fig. 4b the result of a theoretical analysis based on the path integral approach, which is well suited for this Fresnel-like diffraction problem.

In addition to the existence of a minimum width $\Delta T$, which is a direct consequence of the time-uncertainty relation, there are two other signatures of the temporal diffraction phenomenon in the set of data presented here. First, side bands appear on the time-of-flight data of Fig. 4a (see in particular the inset, in which the data have been averaged 850 times and smoothed over every 3 consecutive data points). Because of the convolution of the signal with the detecting pulse $P_{3}$, they are not as visible as in spatial diffraction experiments (see e.g. [6] for neutron diffraction). Second, for large $\tau$, the width $\Delta T$ is found to be smaller by $\approx 10 \mu$ s than the classical prediction (broken line in Fig. $4 \mathrm{~b}$ ). This originates from the temporal equivalent, on each side of $P_{2}$, of the well-known edge diffraction phenomenon [7].

\section{A temporal Young slit interferometer for atoms}

The time-diffracted atomic wave packet produced in the experiment described above can now be used as a source for an interferometry experiment. In this purpose we have designed a temporal Young slit interferometer by splitting $P_{2}$ into two pulses $P_{2 \mathrm{a}}$ and $P_{2 \mathrm{~b}}$ separated by a variable duration $\tau^{\prime}$. This is an analog for de Broglie waves of the Sillitto-Wykes photon experiment $[8]^{\dagger}$. The interference occurs between the paths $P_{1}-P_{2 \mathrm{a}}-P_{3}$ and $P_{1}-P_{2 \mathrm{~b}}-P_{3}$, as in a Young double slit experiment [10]. Figure 5a presents the time-of-flight distribution obtained with $\tau^{\prime}=40 \mu \mathrm{s}$. The measured interference profile between the paths $P_{1}-P_{2 \mathrm{a}}-P_{3}$ and $P_{1}-P_{2 \mathrm{~b}}-P_{3}$ is in good agreement with the one calculated using the path integral approach, shown in a continuous line, which takes into account the finite width of the temporal slits.

The relative phase between the two arms of this interferometer can be easily scanned by changing the effective height of the mirror for $P_{2 \mathrm{~b}}$, with respect to the mirror height for $P_{2 \mathrm{a}}$. This is done by changing the mirror intensity $I$ by a quantity $\delta I$ during the pulse $P_{2 \mathrm{~b}}$, which displaces the atom turning point by

$$
\delta z=(2 \kappa)^{-1} \delta I / I,
$$

and modifies the path length of $P_{1}-P_{2 \mathrm{~b}}-P_{3}$ by $2 \delta z$. A phase shift of $\pi$ between the two paths is obtained for $\delta z=\Lambda_{\mathrm{dB}} / 4$, where $\Lambda_{\mathrm{dB}}=h / m g T=12 \mathrm{~nm}$ is the de Broglie wavelength of the atoms incident on the mirror. In our experimental condition, this corresponds to $\delta I / I=0.03$. The interference profile obtained in this situation is shown in Fig. 5b. As expected, the central fringe then corresponds to a destructive interference.

\footnotetext{
†The extension of Ref. [8] to neutrons has been discussed theoretically in Ref. [9].
} 

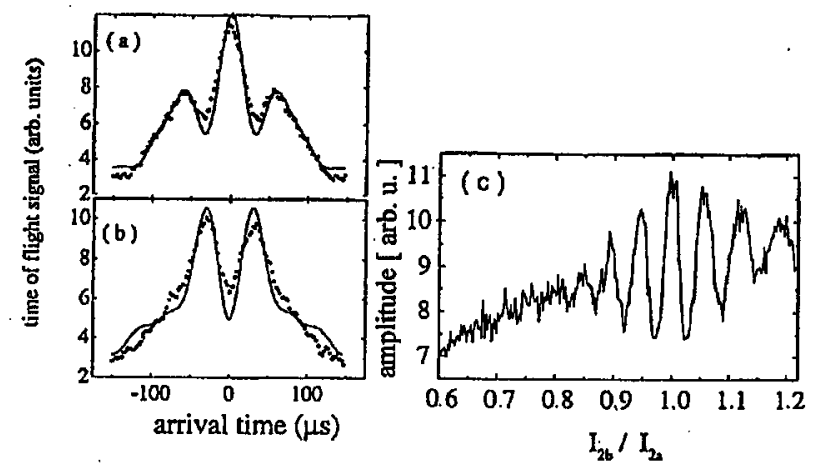

Fig. 5. Experimental $(\bullet)$ and calculated (continuous line) time-of-flight signals obtained in a temporal Young-slit configuration, in which the pulse $P_{2}$ is split into two pulses $P_{2 \mathrm{a}}$ and $P_{2 \mathrm{~b}}$ separated by a duration $\tau^{\prime}=40 \mu \mathrm{s}$. (a) Equal mirror intensity for $P_{2 \mathrm{a}}$ and $P_{2 b}$. (b) Phase shift of $\pi$ between the two paths of the interferometer, obtained experimentally using a $3 \%$ reduction of the evanescent wave intensity during $P_{2 \mathrm{~b}}$. (c) Amplitude of the central fringe of the temporal Young-slit interferometer as a function of the evanescent wave intensity $I_{2 \mathrm{~b}}$ during the pulse $P_{2 \mathrm{~b}}$. The intensity $I_{2 \mathrm{a}}$ during the pulse $P_{2 \mathrm{a}}$ is constant. The maxima correspond to a path difference between the two arms multiple of $\Lambda_{\mathrm{dB}} / 2=6 \mathrm{~nm}$.

Finally, Fig. $5 \mathrm{c}$ gives the interference signal obtained with the detecting pulse $P_{3}$ set on the central fringe $(t=5 T)$, when the intensity for $P_{2 \mathrm{~b}}$ is scanned. Several extrema are visible and each maximum corresponds to constructive interference which occurs when the turning point in $P_{2 \mathrm{~b}}$ is displaced by a multiple of $\Lambda_{\mathrm{dB}} / 2$. The reduction of the contrast as $\delta I$ increases is due to the dispersion in $\Lambda_{\mathrm{dB}}$ resulting from the use of a sequence of several pulses for $P_{1}, P_{2}, P_{3}$. Also the van der Waals interaction between the bouncing atoms and the dielectric modifies the simple prediction (2), so that the phase shift actually varies with the distance between the turning point of the atomic trajectory and the dielectric surface. It therefore depends on the atomic transverse position in the Gaussian evanescent wave. This latter experiment can be used for a measurement of the van der Waals interaction between the atom and the dielectric surface ${ }^{\ddagger}$, by analyzing precisely the variation of the phase difference between the two paths, as the turning point of $P_{2 \mathrm{~b}}$ gets closer to the dielectric.

\section{Phase modulation of matter waves.}

Atomic mirrors with evanescent waves can be used in a way which is richer than simply blocking and unblocking an atomic beam. For instance by modulating the evanescent wave intensity, we can mimic a vibrating mirror. When atoms prepared in a state of well-defined total energy are reflected from such a vibrating mirror, the associated matter waves are phase modulated. The resulting beam

$\ddagger$ A meaurement of this van der Waals interaction using an atomic mirror is reported in Ref. [11]. 
then consists of a "carrier" plus various side bands corresponding to de Broglie waves propagating at different velocities [12].

To observe such a phenomenon [13], we prepare as before cesium atoms in the magneto-optical trap of the lower chamber and we release them. They form a cold "beam", moving vertically downwards. They are velocity-selected using a sequence of two short pulses of the EW intensity (Fig. 6a). Each pulse has a duration $\tau=0.4 \mathrm{~ms}$ and their centers are separated by $T=52 \mathrm{~ms}$, with the first pulse centered $T / 2=26 \mathrm{~ms}$ after the atoms have been released from the MOT. The velocity distribution which is thus selected is triangular, centered at $v_{i}=g T / 2=25.5 \mathrm{~cm} / \mathrm{s}$, with a width (HWHM) $\Delta v=g \Delta T / 4=0.098 \mathrm{~cm} / \mathrm{s}$. Note that this value of $\tau$ is large enough for a classical reasoning to be valid; in other words the time diffraction phenomenon described in Sec. 3 is negligible in these conditions.
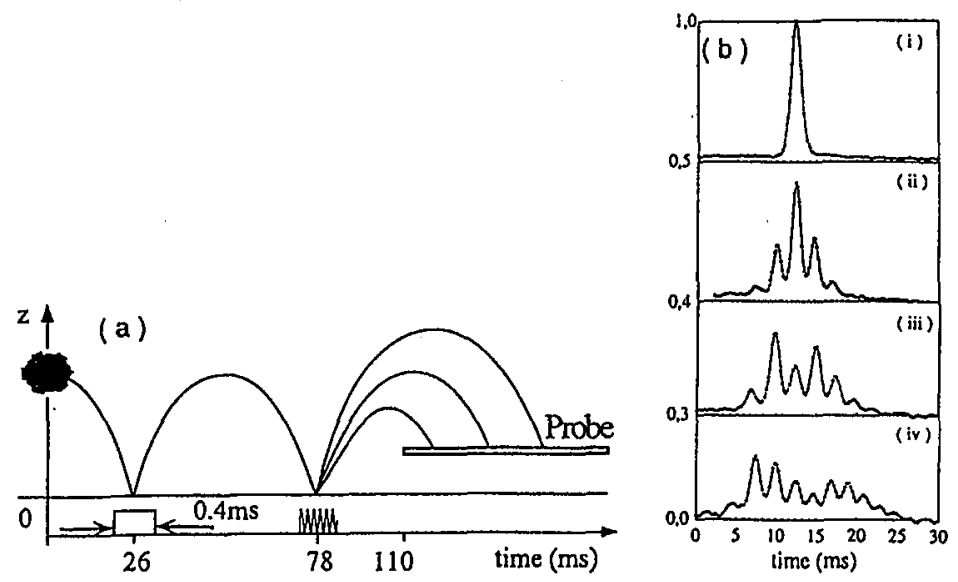

Fig. 6. Phase modulation of de Broglie waves (a) Atoms with a well-defined energy are selected using a two-pulse technique. The modulation of the EW during the second pulse introduces sidebands onto the de Broglie waves. These are detected by their time of flight to a probe beam introduced after the second pulse. (b) Time-of-flight signals. (i) Non-modulated potential; (ii)-(iv) modulated potential, with frequency $\nu=950 \mathrm{kHz}$ (ii), $880 \mathrm{kHz}$ (iii), and $800 \mathrm{kHz}$ (iv).

The atomic kinetic energy is $E / h=10.42 \pm 0.03 \mathrm{MHz}$ at the mirror surface. During the second pulse, the amplitude of the light field is modulated at a chosen frequency between 0 and $2 \mathrm{MHz}$. The presence of several frequency components in the reflected atomic beam is deduced by recording the time taken for the beam to rise and then fall back down to a fixed "probe" height in the earth's gravitational field. Since the matter waves' group velocity (at any given height) depends on their frequency, the negative-order side bands arrive first at the probe, followed by the carrier, followed by the positive side bands. The presence of these side bands clearly demonstrates the quantum nature of the phenomenon, since they could not appear in the reflection of a classical point-like particle on a modulated potential. 
They demonstrate also a direct transfer of a precisely synthesised r.f. frequency onto the atomic motion.

The time-of-flight (TOF) signal shown in Fig. $6 \mathrm{~b}(\mathrm{i})$ was obtained with a non-modulated mirror $(\epsilon=0)$. It represents the average of 1000 shots, with $\approx 4000$ atoms contributing per shot. The TOF spectra $6 \mathrm{~b}$ (ii-iv) were obtained with various modulation frequencies $\nu$ with a modulation depth $\epsilon=0.82$. We have measured from these spectra the positions $t_{n}$ of the side bands, from which we derive the corresponding energy transfers. These agree with the theoretical predictions. From our measurements, we deduce $h / M^{2}=3.14 \pm 0.02 \times 10^{-11} \mathrm{~s}^{3}$, to be compared with the expected value $3.120 \times 10^{-11} \mathrm{~s}^{3}$, using the local $g=9.81 \mathrm{~m} / \mathrm{s}^{2}$ in Paris.

This device opens up new possibilities for precision experiments in atom optics, in complement to current gratings and slits, since one can take advantage of the fact that frequency and time intervals can be produced more accurately than distance intervals. This feature is now basic to high precision methods in photon optics, such as frequency chains using electro-optics modulators. In neutron optics, direct frequency transfer can be achieved by reflection of a neutron beam off a crystal lattice vibrating at one of its resonant frequencies [14, 15]. For atoms or molecules, most experiments [16] using precise r.f. techniques have relied on the presence of a narrow resonant transition between internal states of the system. By contrast, in our experiment and in a recent experiment performed in Innsbruck [17], demonstrating a frequency shifter for atomic matter waves as a generalization of an acousto-optic shifter for photons, the internal structure of the atom plays no role in defining the modulating frequency, and the latter can be varied continuously.

\section{Dissipation with atomic mirrors}

We have seen that the ability to vary in time the intensity of the evanescent mirror enables us to manipulate the motion of the reflected atoms. Pursuing this theme, we have performed further experiments in which the mirror is used to accelerate or decelerate reflected atoms in a controlled way, thus enabling the atomic trajectories to be focussed [18]. All such experiments are conservative, and so do not permit the brightness of the atomic beams to be increased. Equivalently, the phase space density of the atoms is conserved. However, atomic mirrors, unlike their photonic equivalents, can also provide dissipation. Using a spontaneous emission process during the reflection of the atoms, one can take advantage of the Sisyphus cooling mechanism to reduce their kinetic energy much below the incident one. This idea was first proposed in Ref. [19] and later on investigated theoretically in detail in Ref. [20]. The atomic ground level has to involve at least two states which experience a different and spatially dependent light shift by the evanescent wave. A spontaneous Raman transition from the most shifted state to the other one may occur during the bouncing process, which leads to a reduction of the atomic kinetic energy.

A first experimental evidence for such a cooling process was reported in Ref. [21]. A thermal atomic beam was sent at grazing incidence onto an atomic mirror, and a non-specular reflected beam was observed, corresponding to a decrease in the atomic kinetic energy due to the Sisyphus process. A good agreement 
between the experimental results and a simple theoretical model was obtained concerning the average energy loss. We report here on an observation of this cooling process for our laser cooled atoms dropped at normal incidence onto the evanescent wave mirror (for more details, see also [22]).

We model the cesium atoms as a three-level system, with an unstable excited state $e$ and two stable ground states. These two states correspond to the hyperfine ground levels $\left(6 s_{1 / 2}, F_{\mathrm{g}}=3\right.$ and $\left.F_{\mathrm{g}}=4\right)$ of the cesium atom separated by $\Delta=$ $2 \pi \times 9.193 \mathrm{GHz}$. The excited state corresponds to the level $6 p_{3 / 2}$, whose hyperfine structure can be neglected since it is small compared with the laser detunings chosen in the experiment.

The interaction between the atom and the evanescent wave gives rise to a potential which depends on the ground state

$$
\begin{aligned}
& U_{3}(z)=\frac{\hbar \Omega_{0}^{2}}{4 \delta} \exp (-2 \kappa z), \\
& U_{4}(z)=\frac{\hbar \Omega_{0}^{2}}{4(\delta+\Delta)} \exp (-2 \kappa z)=\frac{\delta}{\delta+\Delta} U_{3}(z),
\end{aligned}
$$

where $\delta=\omega_{\mathrm{L}}-\omega_{3}$ is the detuning between the laser frequency and the atomic resonance corresponding to the transition $6 s_{1 / 2}, F_{\mathrm{g}}=3 \leftrightarrow 6 p_{3 / 2}$. The potential $U_{4}(z)$ is proportional to $U_{3}(z)$, but weaker.

Consider an atom in state $F_{g}=3$ with kinetic energy $E_{i}=m v_{0}^{2} / 2$ entering into the wave. It experiences the repulsive potential, so that its kinetic energy decreases, whereas its potential energy increases. If we choose the intensity and the detuning such that the spontaneous emission probability per bounce is much smaller than unity, the spontaneous emission process, if it occurs, will preferentially take place in the vicinity of the classical turning point $z_{0}$, given by $E_{i}=U_{3}\left(z_{0}\right)$. The atom may then fall back to either one of the two ground states.

If it ends up in $F_{\mathrm{g}}=3$, it will continue its way, without being perturbed, if we neglect the atomic recoil during absorption and emission. However, the atom may also fall into $F_{\mathrm{g}}=4$. While the kinetic energy remains constant during this transition, the atom now experiences the potential $U_{4}(z)$ which is weaker than $U_{3}(z)$. After the bounce, the atomic kinetic energy $E_{\mathrm{f}}=m v_{\mathrm{f}}^{2} / 2$ is thus smaller than the initial one $[19,20]$. The loss of potential energy is maximal when the scattering process occurs at $z_{0}$. The final energy in this case is given by

$$
E_{\mathrm{f}}^{\min }=E_{i} \frac{\delta}{\delta+\Delta}
$$

The atoms are prepared in the MOT located $3 \mathrm{~mm}$ above the prism as described above. We then block the repumping laser at a time referred to as $t=0$ in the following. Consequently almost all atoms are optically pumped into the $F_{\mathrm{g}}=3$ ground state in which they no longer interact with the light and fall under the influence of gravity. At $t=6 \mathrm{~ms}$, we also block the main lasers resonant with the $F_{\mathrm{g}}=4 \leftrightarrow F_{\mathrm{e}}=5$ transition.

If the Sisyphus transition occurs, it changes the velocity of the reflected atoms. We analyze the energy distribution of these atoms by a TOF starting at $t=43 \mathrm{~ms}$ (Fig. 7). We record the absorption of a horizontal probe laser beam resonant with the $F_{\mathrm{g}}=4 \leftrightarrow F_{\mathrm{e}}=5$ transition. The probe is centered $450 \mu \mathrm{m}$ 


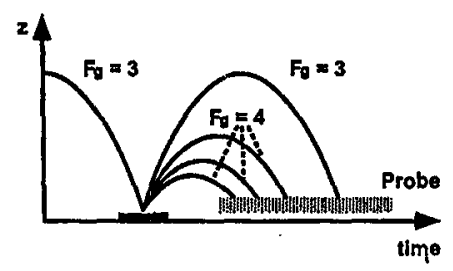

Fig. 7. A fraction of ground state $\left(F_{\mathrm{g}}=3\right)$ atoms bouncing on the mirror can undergo a Sisyphus transition towards $F_{\mathrm{g}}=4$ in the evanescent wave. The energy loss results in a shorter arrival time in the probe beam.

above the evanescent wave mirror. It may be t.ixed with a repumping beam $F_{\mathrm{g}}=3 \leftrightarrow F_{\mathrm{e}}=4$, so that we can choose between the detection of atoms either in $F_{\mathrm{g}}=4$ or in both hyperfine states. We can therefore determine the proportion of atoms undergoing the Sisyphus transition.

Figure 8a gives a typical atomic TOF curve. It shows the probe absorption as a function of time $t$. The bouncing period for atoms in state $F_{\mathrm{g}}=3$, which undergo a specular reflection, is $53 \mathrm{~ms}$. These atoms cross the probe laser mixed with the repumping beam at $t=83 \mathrm{~ms}$. Atoms undergoing a Sisyphus transition loose energy during the reflection and leave the mirror at a smaller velocity and with a shorter bouncing period. They arrive first at the detection laser and they give rise to a corresponding broad peak of low height, whose maximum is located around the arrival time $t_{\mathrm{Sis}}=53 \mathrm{~ms}$. The signal was recorded using a mirror detuning of $\delta=2 \pi \times 3000 \mathrm{MHz}$ and a repumping laser was introduced in the probe beam so that both ground hyperfine levels were detected.

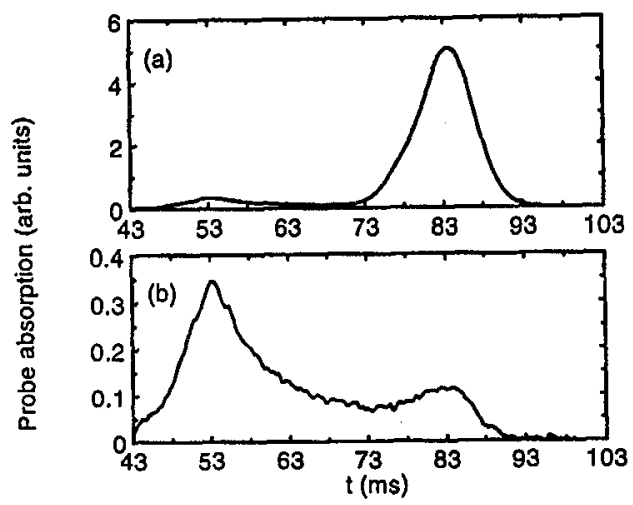

Fig. 8. TOF signals showing a Sisyphus cooling in a single bounce: (a) The atoms are detected both in $F_{\mathrm{g}}=3$ and $F_{\mathrm{g}}=4$ using a probe beam including a repumping laser. The slowed atoms arrive first (peak centred at $t_{\mathrm{Sis}}=53 \mathrm{~ms}$ ) followed by the uncooled atoms (peak centred at $t=83 \mathrm{~ms}$ ). (b) Same experiment without a rempumping beam in the probe; only the cooled atoms (in $F_{\mathrm{g}}=4$ ) are detected. 
In order to prove that this signal corresponds to atoms undergoing a Sisyphus process, we repeated the measurement detecting only atoms in the state $F_{\mathrm{g}}=4$, i.e. without repumping laser. The result is presented in Fig. $8 \mathrm{~b}$. The peak previously detected at $83 \mathrm{~ms}$, which corresponds to atoms in state $F_{\mathrm{g}}=3$, nearly disappears, whereas the earlier observed signal is unchanged. The atoms corresponding to this broad peak maximum at $t_{\mathrm{Sis}}$ are thus in state $F_{\mathrm{g}}=4$.

This elementary Sisyphus process is a convenient tool to accumulate a large number of atoms in a restricted domain of space, increasing therefore the quantum degeneracy of the gas. As pointed out in Ref. [19] and [21], and as shown experimentally very recently in Ref. [23], the repetition of such processes, alternated with repumping phases transferring the atoms back to $F_{\mathrm{g}}=3$, should lead to an atomic gas with a kinetic energy of a few recoil energies $\hbar^{2} k^{2} / 2 m$ only, where $\hbar k$ is the momentum of a single photon.

This Sisyphus process can also be used to populate efficiently the ground state of a potential confining the atoms in the vicinity of the dielectric prism, achieving thus a quasi bidimensional gas [24-26]. This could provide an efficient way to prepare a $2 \mathrm{D}$ gas with a high quantum degeneracy.

We acknowledge stimulating discussions and general encouragement from the E.N.S. laser cooling group. M.A. acknowledges financial support by the Alexander von Humboldt Foundation. This work has been partially supported by DRET, CNRS, Collège de France, DRED and the T.M.R. program of the European Community (contract FMRX-CT960002).

\section{References}

[1] R.J. Cook, R.K. Hill, Opt. Commun. 43, 258 (1982).

[2] V.I. Balykin, V.S. Letokhov, Yu.B. Ovchinnikov, A.I. Sidorov, Sov. Phys. JETP Lett. 45, 353 (1987); Phys. Rev. Lett. 60, 2137 (1988).

[3] C.G. Aminoff, A. Steane, P. Bouyer, P. Desbiolles, J. Dalibard, C. Cohen-Tannoudji, Phys. Rev. Lett. 71, 3083 (1993).

[4] M. Moshinski, Phys. Rev. 88, 625 (1952); A.S. Gerasimov, M.V. Kazarnovskii, Sov. Phys. JETP 44, 892 (1976); J. Felber, G. Müller, R. Gälher, R. Golub, Physica B 162, 191 (1990); A. Zeilinger, C. Brukner, unpublished.

[5] P. Szriftgiser, D. Guéry-Odelin, M. Arndt, J. Dalibard, Phys. Rev. Lett. 77, 4 (1996).

[6] R. Gähler, A. Zeilinger, Am. J. Phys. 59, 316 (1991).

[7] M. Born, E. Wolf, Principles of Optics, 6th ed., Pergamon, New York 1980, p. 433.

[8] R.M. Sillitto, C. Wykes, Phys. Lett. A 39, 333 (1972).

[9] H.R. Brown, J. Summhammer, R.E. Callaghan, P. Kaloyerou, Phys. Lett. A 163, 21 (1992).

[10] O. Carnal, J. Mlynek, Phys. Rev. Lett. 21, 2689 (1991); F. Shimizu, K. Shimizu, H. Takuma, Phys. Rev. A 46, R17 (1992).

[11] A. Landragin; J.-Y. Courtois, G. Labeyrie, N. Vansteenkiste, C.I. Westbrook, A. Aspect, Phys. Rev. Lett. 77, 1464 (1996).

[12] C. Henkel, A.M. Steane, R. Kaiser, J. Dalibard, J. Phys. II (France) 4, 1877 (1994). 
[13] A. Steane, P. Szriftgiser, P. Desbiolles, J. Dalibard, Phys. Rev. Lett. 74, 4972 (1995).

[14] W.A. Hamilton, A.G. Klein, G.I. Opat, P.A. Timmins, Phys. Rev. Lett. 58, 2770 (1987).

[15] J. Felber, Ph.D., Technischen Universität, Munich 1994, unpublished; J. Felber, R. Gähler, C. Rausch, R. Golub, Phys. Rev. A 53, 319 (1996).

[16] Atom Interferometry, Ed. P. Berman, Academic Press, New York 1996 and references therein.

[17] S. Bernet, M. Oberthaler, R. Abfalterer, J. Schmiedmayer, A. Zeilinger, Phys. Rev. Lett. 77, 5160 (1996).

[18] M. Arndt, P. Szriftgiser, J. Dalibard, A. Steane, Phys. Rev. A 53, 3369 (1996).

[19] K. Helmerson, S. Rolston, L. Goldner, W.D. Phillips, poster at the Workshop on Optics and Interferometry with Atoms, Insel Reichenau (Germany) 1992, unpublished; Quantum Electronics and Laser Science Conference, 1993, OSA Technical Digest Series, Vol. 12, O.S.A., Washington DC 1993, p. 209.

[20] J. Söding, R. Grimm, Yu.B. Ovchinnikov, Opt. Commun. 119, 652 (1995).

[21] Yu.B. Ovchinnikov, D.V. Laryushin, V.I. Balykin, V.S. Letokhov, JETP Lett. 62, 113 (1995).

[22] P. Desbiolles, M. Arndt, P. Szriftgiser, J. Dalibard, Phys. Rev. A 54, 4292 (1996).

[23] Yu.B. Ovchinnikov, I. Manek, R. Grimm, unpublished.

[24] P. Desbiolles, J. Dalibard, Opt. Commun. 132, 540 (1996).

[25] M. Reynolds, J. Walraven, private communication (November 1994).

[26] T. Pfau, J. Mlynek, OSA Trends in Optics and Photonics Series on Bose-Einstein Condensation 7, 33 (1996). 